\title{
Hydrogen Spectroscopy with a Lamb-shift Polarimeter
}

\section{An Alternative Approach Towards Anti-Hydrogen Spectroscopy Experiments}

\author{
M. P. Westig ${ }^{1,2}$, R. Engels ${ }^{2}$, K. Grigoryev ${ }^{2,3}$, M. Mikirtytchiants ${ }^{2,3}$, F. Rathmann ${ }^{2}$, H. Paetz gen. Schieck ${ }^{4}$, G. Schug ${ }^{2}$, \\ A. Vasilyev ${ }^{3}$, and H. Ströher ${ }^{2}$ \\ 1 I. Physikalisches Institut, Universität zu Köln, 50937 Köln, Germany \\ 2 Institut für Kernphysik and Jülich Center for Hadron Physics, Forschungszentrum Jülich GmbH, 52425 Jülich, Germany \\ 3 Petersburg Nuclear Physics Institute, 188300 Gatchina, Russia \\ 4 Institut für Kernphysik, Universität zu Köln, 50937 Köln, Germany
}

Received: date / Revised version: date

\begin{abstract}
A Lamb-shift polarimeter, which has been built for a fast determination of the polarization of protons and deuterons of an atomic-beam source and which is frequently used in the ANKE experiment at COSY-Jülich, is shown to be an excellent device for atomic-spectroscopy measurements of metastable hydrogen isotopes. It is demonstrated that magnetic and electric dipole transitions in hydrogen can be measured as a function of the external magnetic field, giving access to the full Breit-Rabi diagram for the $2^{2} S_{1 / 2}$ and the $2^{2} P_{1 / 2}$ states. This will allow the study of hyperfine structure, $g$ factors and the classical Lamb shift. Although the data are not yet competitive with state-of-the-art measurements, the potential of the method is enormous, including a possible application to anti-hydrogen spectroscopy.
\end{abstract}

PACS. 32.10.Fn Fine and hyperfine structure $-32.60 .+\mathrm{i}$ Zeeman and Stark effects $-37.20 .+\mathrm{j}$ Atomic and molecular beam sources and techniques

The spectrum of atomic hydrogen $(\mathrm{H})$, unravelled with ever increasing precision, has led to fundamental understanding of the underlying structure and dynamics of the simplest atom. Today, both experiment (e.g., two-photon laser spectroscopy [1,2]) and theory [3] have achieved an impressive level of accuracy in the determination of the energy levels. Additional details and further discoveries may come from either advancing existing techniques or by applying new methods.

A possible new approach to performing spectroscopy experiments on hydrogen isotopes is based on a Lambshift polarimeter which would allow measurements of the full Breit-Rabi diagram of the first excited state of $\mathrm{H}$ with $j=1 / 2$ (see Fig. 1). Precision data currently exist only for weak magnetic fields of a few Gauss for the ground state 4. For the metastable state, the crossing of the $2^{2} S_{1 / 2}$ and $2^{2} P_{1 / 2}$ states ( $\beta-$ e crossing of Fig. 11) at field strengths of about $570 \mathrm{G}$ was investigated long ago 5 . In addition to testing the recent relativistic theory of the Zeeman splitting of the first excited state 6. 7], systematic Breit-Rabi diagram measurements can also be used to extract $g_{j}\left(\mathrm{H}, 2^{2} S_{1 / 2}\right), g_{j}\left(\mathrm{H}, 2^{2} P_{1 / 2}\right)$ and the nuclear $g$ factor. The most recent determination of a hydrogen $g_{j}$ factor for the ground state was performed by Tiedeman and Robinson 8. Mass independent terms of quantum electrodynamics could be tested to a high degree and a confirmation of the $\alpha^{3}$ radiative correction has been achieved. In order to test, e.g., bound-state quantum electrodynamic corrections of the order of $\alpha / \pi$ for the $g_{j}$ factor, a precision of $1 \mathrm{ppb}$ and better is needed for the hydrogen atom in the ground state 9. An even better precision would be needed for the first excited state. By putting the hydrogen atom in an external magnetic field, the independence of the hyperfine structure to such conditions can be tested through a combination of measurements of transitions between $2^{2} S_{1 / 2}$ and $2^{2} P_{1 / 2}$ states (Table 1).

In 1940, Kusch et al. performed spectroscopy experiments on alkaline atoms in a magnetic field with an improved molecular beam resonance method for atoms and determined the hyperfine structure and nuclear moments from measurements of the Breit-Rabi diagram 10. Though Rabi realized that this method should also be applicable to excited atomic states [1], it seems never to have been exploited before. The measurements of Lundeen and Pipkin 12, 13 provide the best direct determinations of the classical Lamb shift. Their experiment used transitions between the hyperfine structure of the $2^{2} S_{1 / 2}$ and $2^{2} P_{1 / 2}$ states. In contrast, we present an enhanced experimental method to measure transitions between single Zeeman components of the hyperfine structure with a spin-filtering technique. Besides the hyperfine structure and the classical Lamb shift, the measurement of these transitions will allow to determine the Breit-Rabi diagram of the $2^{2} S_{1 / 2}$ and the $2^{2} P_{1 / 2}$ state as is shown in this paper. 


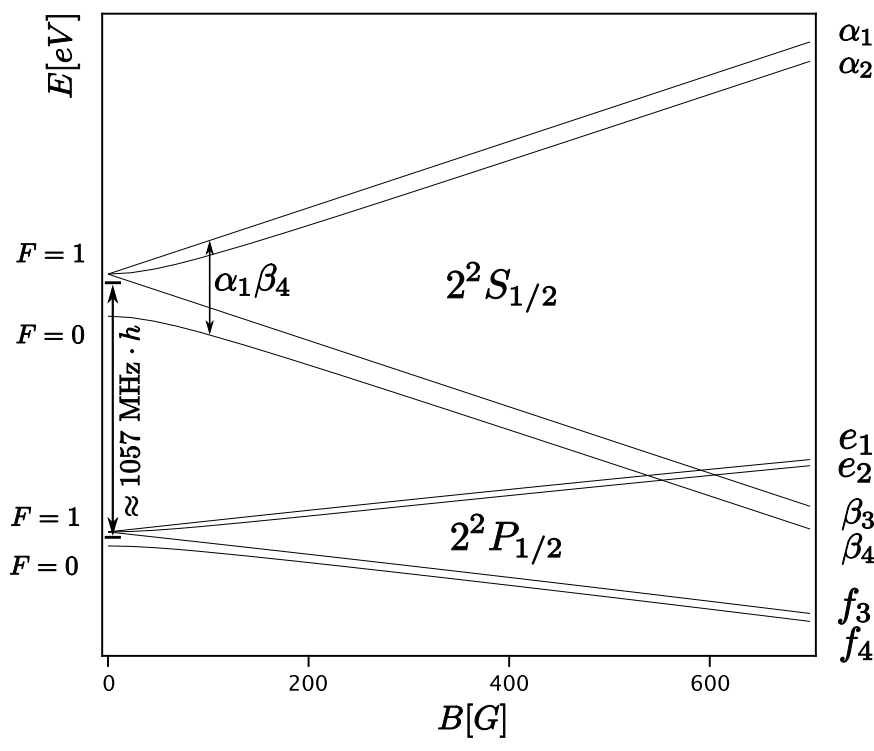

\begin{tabular}{l|l|l} 
State & Zeeman Region & \multicolumn{1}{|c}{ Paschen-Back Region } \\
\hline$\alpha_{1}$ & $\left|F=1, m_{F}=1\right\rangle$ & $\left|m_{j}=1 / 2, m_{I}=1 / 2\right\rangle$ \\
$\alpha_{2}$ & $|1,0\rangle$ & $f^{+}|1 / 2,-1 / 2\rangle+f^{-}|-1 / 2,1 / 2\rangle$ \\
$\beta_{3}$ & $|1,-1\rangle$ & $|-1 / 2,-1 / 2\rangle$ \\
$\beta_{4}$ & $|0,0\rangle$ & $f^{-}|1 / 2,-1 / 2\rangle-f^{+}|-1 / 2,1 / 2\rangle$
\end{tabular}

Fig. 1. Breit-Rabi diagram of $\mathrm{H}$ for the first excited state with $j=1 / 2$ 6. 7. The classical Lamb shift of $1057 \mathrm{MHz}$ and a magnetic dipole transition from the state $\alpha_{1}$ into $\beta_{4}$ are indicated. The table indicates the definitions for the Zeeman and the Paschen-Back regions for the $\alpha$ - and $\beta$-states, where $f^{ \pm}=$ $1 / \sqrt{2} \sqrt{1 \pm a(B)}$ are the critical field parameters describing the transition between them and $a(B)=B /\left(B_{C} \sqrt{1+\left(B / B_{C}\right)^{2}}\right)$ with the critical field strength $B_{C}=\Delta E_{\mathrm{HFS}} / 2 \mu_{B}$ where $\Delta E_{\mathrm{HFS}}$ denotes the energy separation of the hyperfine states of the $2^{2} S_{1 / 2}$ state and $\mu_{B}$ is the Bohr magneton. Corresponding definitions apply for the state $2^{2} P_{1 / 2}$.

At the Forschungszentrum Jülich, we have built an atomic-beam source to produce beams of polarized hydrogen and deuterium [14 for use as internal targets in experiments at the cooler storage ring COSY [15]. The atomic-beam source has been complemented by a Lambshift polarimeter [16, 17] for the fast on-line determination of the target polarization. When not required for this purpose, we have started to use the Lamb-shift polarimeter - atomic-beam source combination to study atomic hydrogen spectroscopy in a dedicated laboratory setup. The results of a proof-of-principle experiment described here demonstrate that a metastable beam of atoms produced in a well-defined Zeeman state $\left(\alpha_{1}\right.$ or $\alpha_{2}$ of Fig. 11, see also Fig. 2) by the Lamb-shift polarimeter, will be an excellent tool for precision hydrogen spectroscopy.

We start by briefly recalling the operating principles of the atomic-beam source and the Lamb-shift polarimeter. The atomic-beam source which might be used in a future setup provides a cold atomic beam emerging from a dissociator and a cooled nozzle. Atoms with a definite total an-

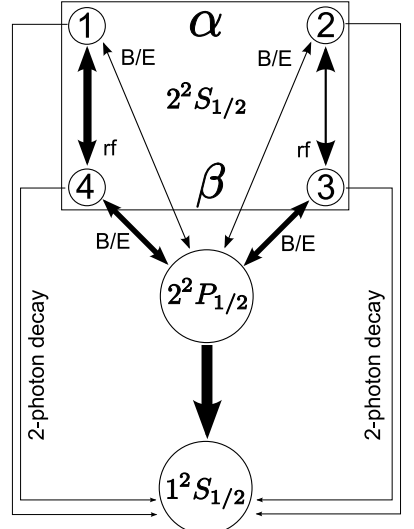

(a)

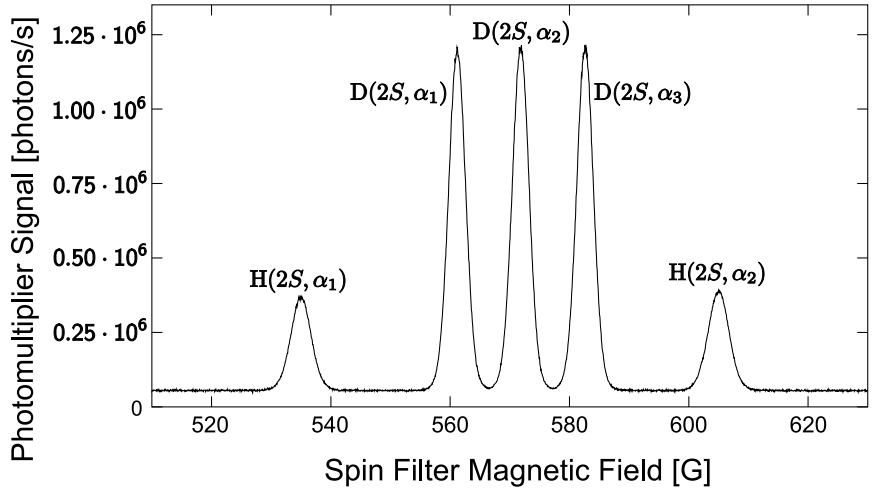

(b)

Fig. 2. (a) Principle of the spin-filtering technique for metastable hydrogen. In the absence of magnetic fields, $2^{2} S_{1 / 2}$ states can decay to the $1^{2} S_{1 / 2}$ state by 2 -photon emission. However, the simultaneous interaction with a magnetic field $B$, an electric field $E$ and a radio-frequency $(r f)$ field, allows one to select, e.g., state $\alpha_{1}$ : by tuning the magnetic field to $535 \mathrm{G}$, state $\beta_{4}$ intersects with $e_{1}$ while $\beta_{3}$ is very close to the shortlived $2^{2} P_{1 / 2}$ states. An electric field mixes $\beta_{3}$ and $\beta_{4}$ with the $2^{2} P_{1 / 2}$ state with subsequent decay to the ground state. The radio-frequency field strongly couples $\alpha_{1}$ and $\beta_{4}$ (thick arrow), while it weakly connects $\alpha_{2}$ and $\beta_{3}$ (thin arrow). This leads to an emptying of $\alpha_{2}$ by means of $\beta_{3}$ while $\alpha_{1}$ stays in oscillation with $\beta_{4}$. Turning off the radio-frequency field, residual atoms in the state $\beta_{3}$ and $\beta_{4}$ are quenched into the ground state and only atoms in state $\alpha_{1}$ are left in the beam. The same principle applies to the $\alpha_{2}$ state and analogously to the deuterium atom. (b) Lyman- $\alpha$ spectrum for metastable hydrogen and deuterium as a function of the magnetic field in the spin filter after optimization of intensity and state separation. The spectrum has been obtained by a photomultiplier on top of the transition unit (see Fig. 3(a) . For hydrogen, states $\alpha_{1}$ and $\alpha_{2}$ can be selected by tuning the magnetic field to $535 \mathrm{G}$ and $605 \mathrm{G}$, respectively. For deuterium, the field strengths are $B=565 \mathrm{G}\left(\alpha_{1}\right), 575 \mathrm{G}\left(\alpha_{2}\right)$ and $585 \mathrm{G}\left(\alpha_{3}\right)$.

gular momentum are filtered by a Stern-Gerlach assembly of sextupole magnets and specific hyperfine states are then selectively populated by radio-frequency units. The Lambshift polarimeter (see Fig. 31) ionizes the atomic beam in 


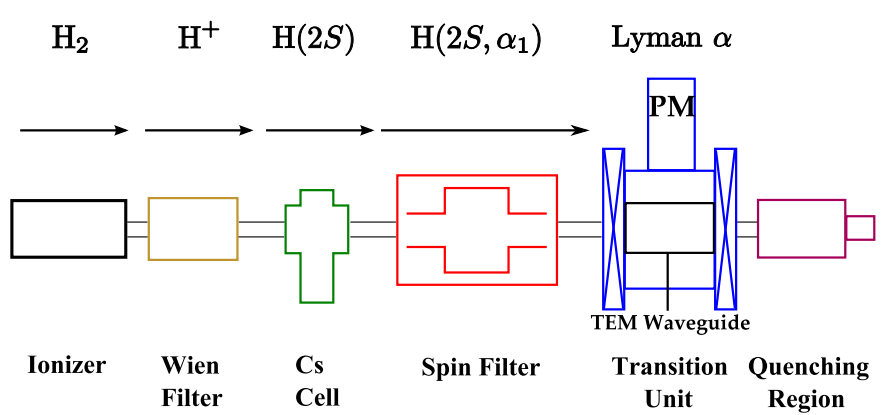

(a)

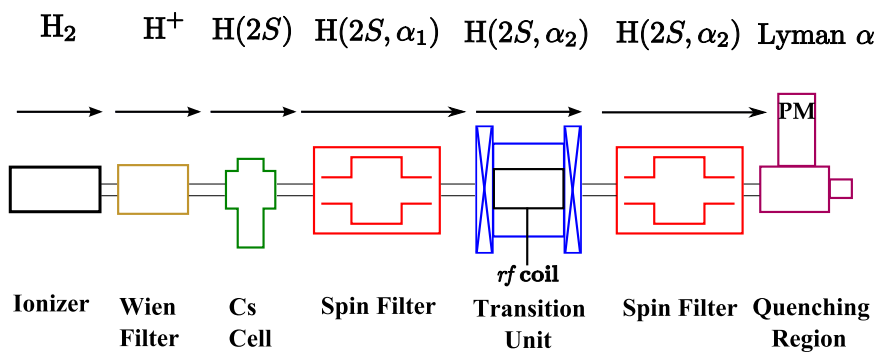

(b)

Fig. 3. (color on-line) (a) Lamb-shift polarimeter setup used for the measurements of electric dipole transitions with atoms in the $\alpha_{1}$ state. (b) Shows the changes necessary for a possible future experiment to measure magnetic dipole transitions, where atoms in the $\alpha_{1}$ state undergo transitions into the $\alpha_{2}$ state. The position of the photomultiplier will change for measurements of electric dipole and magnetic dipole transitions. The transition unit is surrounded by a pair of Helmholtz coils. A future upgrade would replace the ionizer, the Wien filter and the cesium cell by a cold excited atomic beam (see text).

a strong magnetic field to preserve the nuclear spin. After the metastable state is produced in a cesium cell, a spin filter 18, a quenching region with a static electric field and a photomultiplier, sensitive to $121 \mathrm{~nm}$ Lyman- $\alpha$ radiation, is used to determine the beam polarization through the relative occupation of the hyperfine states [16] (see Fig. 21). In the experiment described below the Lamb-shift polarimeter is used to produce metastable atoms in one single Zeeman state of the hyperfine structure $\left(\alpha_{1}, \alpha_{2}\right.$ and together with a Sona transition $\beta_{3}$ [19], see Fig. 1).

The experimental setup is shown schematically in Fig. 3, where the upper part presents the situation for measuring electric dipole transitions. $\mathrm{H}_{2}$ molecules are first ionized inside an electron-impact ionizer. After passing through a Wien filter, which only transmits protons, the ion beam enters a cesium cell in which metastable hydrogen atoms in the $2^{2} S_{1 / 2}$ state are produced by the charge-exchange reaction $\mathrm{H}^{+}+\mathrm{Cs} \rightarrow \mathrm{H}\left(2^{2} S_{1 / 2}\right)+\mathrm{Cs}^{+}$. The beam energy of $1 \mathrm{keV}$ is a compromise between working at the maximum cross section for the process 20 and minimizing losses due to beam divergence. Subsequently, one of the Zeeman states of the hyperfine structure $\left(\alpha_{1}\right.$ or $\alpha_{2}$ ) is selected in a spin filter (see Fig. 2). Finally, these atoms enter the transition unit with a Lecher TEM (trans-
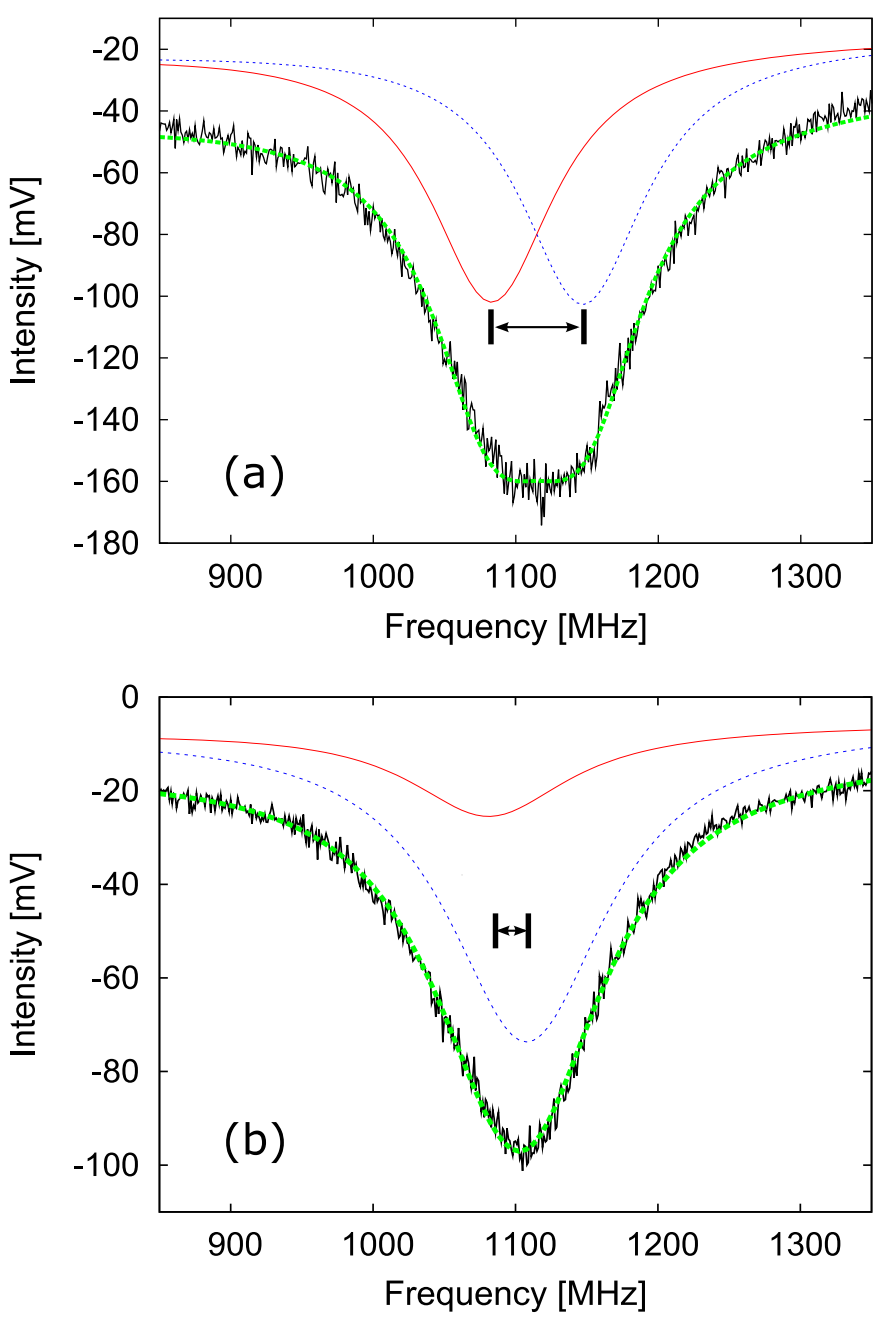

Fig. 4. (color on-line) (a) Result for electric dipole transitions $(\sigma$ transitions) at small external magnetic field strength $(B \approx$ $0 \mathrm{G}$ ) for a beam of atoms in the $\alpha_{1}$ state. The thin black solid line is the measured spectrum while the thick green dashed line is the fit to the data. This is the sum of two single fits shown as thin red solid and dashed blue lines corresponding to the transitions $\alpha_{1} \rightarrow e_{2}, f_{4}$. The spacing between the fitted minima corresponds to the $2^{2} P_{1 / 2}$ hyperfine structure, indicated by the black arrow. (b) Electric dipole transitions ( $\sigma$ transitions) in an external magnetic field of strength $B=26.8(5) \mathrm{G}$ for a beam of atoms in the $\alpha_{2}$ state undergoing transitions into the states $e_{1}$ and $f_{3}$. With increasing magnetic field strength, the transition probability into the state $e_{1}$ clearly decreases (thin red solid line). The black arrow indicates the spacing between the Zeeman states $e_{1}$ and $f_{3}$ (dashed blue line) of the $2^{2} P_{1 / 2}$ hyperfine structure component with total angular momentum quantum number $F=1$.

verse electromagnetic) waveguide, which produces an electric field $\boldsymbol{E}$ parallel to the beam axis.

Figure $3(\mathrm{~b})$ shows the setup for the measurement of magnetic dipole transitions. Here a second spin filter is added between the transition unit and the quenching region, the TEM waveguide is replaced by a radio-frequency 
Table 1. Transition-frequency combinations in hydrogen and deuterium to determine magnetic-field-independent values of the hyperfine structure. Here, e.g., $\alpha_{1} \beta_{4}$ denotes the transition $\alpha_{1} \rightarrow \beta_{4}$. (See also Fig. 11).

\begin{tabular}{ll}
\hline & Hydrogen \\
$f_{\mathrm{HFS}}\left(2^{2} S_{1 / 2}\right)$ & $\alpha_{1} \beta_{4}-\alpha_{2} \beta_{3}$ \\
& $\alpha_{1} \alpha_{2}+\beta_{3} \beta_{4}$ \\
$f_{\mathrm{HFS}}\left(2^{2} P_{1 / 2}\right)$ & $\alpha_{1} f_{4}-\alpha_{2} f_{3}-\alpha_{1} e_{1}+\alpha_{2} e_{2}$ \\
& Deuterium \\
& $\alpha_{1} \beta_{5}-\alpha_{3} \beta_{4}+\alpha_{1} \beta_{6}-\alpha_{2} \beta_{4}$ \\
$f_{\mathrm{HFS}}\left(2^{2} S_{1 / 2}\right)$ & $\alpha_{1} \alpha_{2}+\alpha_{1} \beta_{6}+\beta_{4} \beta_{5}-\alpha_{3} \beta_{4}$ \\
$f_{\mathrm{HFS}}\left(2^{2} P_{1 / 2}\right)$ & $\alpha_{3} e_{3}+\alpha_{2} f_{5}-\alpha_{2} e_{1}-2 \alpha_{3} f_{4}$ \\
& $-\alpha_{1} e_{1}+\alpha_{3} e_{2}+\alpha_{1} f_{6}$ \\
\hline
\end{tabular}

coil, and the photomultiplier is moved to the quenching region.

First data were obtained by measuring electric dipole transitions ( $\sigma$ transitions, $\boldsymbol{B} \perp \boldsymbol{E}$ ) to the $2^{2} P_{1 / 2}$ state inside the TEM waveguide. Because of their short lifetime $\left(10^{-9} \mathrm{~s}\right)$ the atoms will immediately decay to the ground state, emitting Lyman- $\alpha$ photons. These are detected through a hole in the TEM waveguide by a photomultiplier mounted on top of the transition unit. In Fig. 4(a) the result of such a measurement is shown where the external magnetic field was close to zero. The intensity of Lyman- $\alpha$ photons is plotted as a function of the TEM radio frequency. The spectrum, obtained in about 10 min measuring time, contains $\approx 10^{7}$ photons. The peak broadening is due to the superposition of two transitions $\left(\alpha_{1} \rightarrow e_{2}, f_{4}\right.$, Fig. 10) and a two-Lorentzian fit results in the shapes shown in Fig. 4(a). The frequency difference corresponds to the $2^{2} P_{1 / 2}$ hyperfine splitting $f_{\mathrm{HFS}}\left(2^{2} P_{1 / 2}\right)$ for which we obtain $60(2) \mathrm{MHz}$. Although this agrees with theory $(59221.2 \mathrm{kHz}[7]$ ), it has a large error. Our result for the classical Lamb shift measured in the same experiment, 1057(1) MHz agrees with theory and recent experiments [3], but the error is two orders of magnitude too large to be competitive. Possible ways to reduce these errors significantly are discussed below.

In Fig. 4(b) we show a measurement of atoms in the $\alpha_{2}$ state undergoing transitions into the states $e_{1}$ and $f_{3}$ in an external magnetic field of strength $B=26.8(5) \mathrm{G}$. In this case the measured spacing between the states $e_{1}$ and $f_{3}$ is $27(2) \mathrm{MHz}$ compared to the expected value of $24.855 \mathrm{MHz}$ [6, 7]. We have to verify this value with the hardware improvements of our experiment described at the end of this text. Measurements of this kind will allow one to determine the whole Breit-Rabi diagram of the $2^{2} S_{1 / 2}$ and the $2^{2} P_{1 / 2}$ states.

We have also performed measurements with a magnetic field close to zero, oriented parallel to the TEM radio-frequency field (electric dipole transitions, $\pi$ transitions, $\boldsymbol{B} \| \boldsymbol{E})$. In this case, selection rules led to the possible transitions $\alpha_{1} \rightarrow e_{1}$ and $\alpha_{2} \rightarrow e_{2}, f_{4}$. As in the case shown in Fig. 4(b) for atoms in the state $\alpha_{2}$ a decrease in the probability of a transition into state $f_{4}$ is observed if $B$ exceeds the critical field value $\Delta E_{\mathrm{HFS}} / 2 \mu_{B}$, i.e., $a(B) \rightarrow 1$ (see Fig. 1), which is the Paschen-Back effect. These atoms only undergo transitions into state $e_{2}$ and, as a result, a narrower line shape is obtained than that found in Fig. 4(a).

When measuring magnetic dipole transitions, the setup has to be modified, as shown in Fig. 3(b). Behind the first spin filter, atoms in one Zeeman state of the hyperfine structure enter the spectroscopy region where magnetic dipole transitions are induced inside a radio-frequency coil. A second spin filter is subsequently used to check the occurrence of a definite transition between two Zeeman components of the $2^{2} S_{1 / 2}$ hyperfine structure. It is, therefore, tuned to transmit only one particular state.

The photomultiplier on top of the quenching region monitors the occurrence of such a transition during Starkeffect quenching of the residual metastable atomic beam. This scheme is actually very similar to the one suggested by Rabi [1].

Since the electric field is parallel to the beam axis, the wave vector is perpendicular and the longitudinal Doppler effect is suppressed in first order. A signal generator supported by a radio-frequency amplifier was connected to the TEM waveguide where a power meter was used to keep the radio-frequency power level constant. The spectroscopy region itself consists of a commercial stainless steel chamber with a pair of magnetic field coils surrounding the chamber. The spectroscopy region was not shielded against external magnetic fields in these initial measurements.

Although proof-of-principle measurements with the Lamb-shift polarimeter have been achieved, further progress has to be made to ensure that this is a precision spectroscopic tool. Some components of the current setup shown in Fig. 3 must be modified. The stainless steel vacuum chamber in the spectroscopy region should be replaced by an aluminum chamber covered by a $\mu$-metal shielding in order to decrease the influence of external electromagnetic fields on the resonance shape and frequency. Since $\Delta B \approx 0.5 \mathrm{G}$, the uncertainty in the frequency is about $1 \mathrm{MHz}$ and an improved set of magnetic field coils should be implemented in order to reach $\Delta B / B \approx 10^{-4}$. The variations in the controlled radio-frequency power $(\approx 3 \%)$ lead to an uncertainty of approximately $100 \mathrm{kHz}$. With state-of-the-art hardware, the power can be controlled on a $10^{-5}$ level.

In order to extract precise resonance frequencies from our measured spectra, a detailed understanding of the shape is needed. The largest effect arises from the uncertainty principle. Because of the short interaction time of the atoms with the radio-frequency field, a resonance broadening of approximately $45 \mathrm{MHz}$ occurs. For electric dipole transitions, the convolution with the natural resonance width results in a line width of approximately $110 \mathrm{MHz}$, while for magnetic dipole transitions a value of $45 \mathrm{MHz}$ is expected.

The atomic beam has a fixed velocity but, due to scattering and electron capture in the cesium cell, effects of energy straggling of about $1 \%$ have to be taken into account. For measurements in magnetic fields $\leq 100 \mathrm{G}$ errors 
Table 2. Estimate of the projected precision for the $g_{j}$ factor and the hyperfine structure that might be achieved during the further development of the experiment. At this stage of development the Lamb-shift polarimeter will be used together with a separated-oscillatory-field (SOF) setup and with a thermal beam emerging from an atomic-beam source (ABS).

\begin{tabular}{ccccc}
\hline & \multicolumn{2}{c}{$2^{2} S_{1 / 2}$} & \multicolumn{2}{c}{$2^{2} P_{1 / 2}$} \\
& $g_{j}$ factor & HFS & $g_{j}$ factor & HFS \\
SOF & $10^{-7}$ & $100 \mathrm{~Hz}$ & $10^{-6}$ & $1 \mathrm{kHz}$ \\
ABS & $<10^{-8}$ & $10-1 \mathrm{~Hz}$ & - & - \\
\hline
\end{tabular}

for a single measurement of $10 \mathrm{kHz}$ for the $2^{2} S_{1 / 2}$ state and $100 \mathrm{kHz}$ for the $2^{2} P_{1 / 2}$ state are to be expected with this setup. Because the $g_{j}$ factor dominates the slope of the Breit-Rabi diagram, it can be identified with a precision of $10^{-5}$ for $2^{2} S_{1 / 2}$ and $10^{-4}$ for $2^{2} P_{1 / 2}$, respectively. Although such a measurement will allow a precise determination of the Breit-Rabi diagram, the $g_{j}$ factor could only be used to check for relativistic effects. Hence an increase in the magnetic field strength up to $1 \mathrm{~T}$ is necessary to improve the $g_{j}$-factor determination to a precision of $10^{-7}$ for $2^{2} S_{1 / 2}$ and $10^{-6}$ for $2^{2} P_{1 / 2}$.

This level of precision is not sufficient to test boundstate quantum electrodynamic effects of the order of $\alpha / \pi$ for the $2^{2} S_{1 / 2}$ state. To further improve the experimental setup, the possibility of using the separated-oscillatoryfield method 13 will be considered in the design of the new spectroscopy region. Together with a reasonable enlargement of the interaction region, this will decrease the uncertainty of the measurement of magnetic dipole transitions by two orders of magnitude.

As a final step in the improvement, the atomic-beam source could be employed as the source of hydrogen atoms, which are excited, e.g., by electron impact. The reduction of the atomic-beam velocity by more than two orders of magnitude would decrease the error for a single measurement of magnetic dipole transitions by the same amount. By rotating the wave vector, the second order Doppler effect can also be cancelled. The transverse Doppler effect, which increases the resonance frequency, can be estimated by changing the beam energy. The motional Stark effect has to be considered since this also shifts the energy levels.

Measurements can be performed for different magnetic field settings to obtain multiple field-independent values of the $2^{2} S_{1 / 2}$ and $2^{2} P_{1 / 2}$ hyperfine structure which can be averaged to increase the precision. Although the measurement of $f_{\mathrm{HFS}}\left(2^{2} P_{1 / 2}\right)$ is lifetime-limited, high-precision values of $f_{\mathrm{HFS}}\left(2^{2} S_{1 / 2}\right)$ are achievable. The classical Lamb shift can also be extracted from the data with a precision $<10 \mathrm{kHz}$. After implementation of all the discussed improvements, we expect that measurements of $g_{j}$ factors and the hyperfine structure with the accuracies given in Table 2 can be achieved.

A very exciting prospect of the method discussed here is that it could also be applied to beams of anti-hydrogen. Although a similar proposal has been put forward in [21, the method demonstrated in this paper provides addi- tional useful features for such a measurement. During the recombination of an antiproton with a positron, up to $30 \%$ of the anti-H atoms produced end up in the $2^{2} S_{1 / 2}$ state. Due to the detection efficiency of $10^{-3}$, in order to detect 100 photons in the photomultiplier, $3 \times 10^{6}$ anti- $\mathrm{H}$ atoms are necessary. Modifications of the quenching region should increase the detection efficiency by two orders of magnitude, in which case 30000 atoms would be sufficient to obtain the $2^{2} S_{1 / 2}$ hyperfine structure with a precision of $140 \mathrm{kHz}$. Reducing the beam energy down to $1 \mathrm{meV}$ would improve the precision to $5 \mathrm{kHz}$ because the Doppler effect is smaller in this case.

In summary, we have shown that a Lamb-shift polarimeter can be used for atomic spectroscopy. The Lambshift polarimeter measurements of hyperfine structure transitions in atomic hydrogen have not yet reached the level of accuracy that other experiments have achieved for example for the $2^{2} S_{1 / 2}$ state [22, 23, 24, 25]. However, the method will allow the determination of the $2^{2} P_{1 / 2}$ hyperfine structure with up to two orders of magnitude higher precision than most recent results [26. In addition, this method is also applicable to deuterium and might be used in the future for anti-hydrogen.

We are grateful for discussions with Th. Udem, D. L. Moskovkin and D. A. Glazov. We thank other members of IKP-2 of Forschungszentrum Jülich for their help during the measurements and C. Wilkin for carefully reading the manuscript. The support from JCHP-FFE of the Forschungszentrum Jülich is gratefully acknowledged.

\section{References}

1. Th. Udem, A. Huber, B. Gross, J. Reichert, M. Prevedelli, M. Weitz, and T. W. Hänsch, Phys. Rev. Lett. 79, 2646 (1997).

2. A. Huber, Th. Udem, B. Gross, J. Reichert, M. Kourogi, K. Pachucki, M. Weitz, and T. W. Hänsch, Phys. Rev. Lett. 80, 468 (1998).

3. K. Pachucki, J. Phys. B: At. Mol. Opt. Phys. 29, 177 (1996).

4. F. Schmidt-Kaler, D. Leibfried, S. Seel, C. Zimmermann, W. König, M. Weitz, and T. W. Hänsch, Phys. Rev. A 51, 2789 (1995).

5. W. E. Lamb, Jr. and R. C. Retherford, Phys. Rev. 81, 222 (1951).

6. D. L. Moskovkin and V. M. Shabaev, Phys. Rev. A 73, 052506 (2006).

7. D. L. Moskovkin and D. A. Glazov (2007), private comm.

8. J. S. Tiedeman and H. G. Robinson, Phys. Rev. Lett. 39, 602 (1977).

9. Th. Beier, I. Lindgren, H. Persson, S. Salomonson, P. Sunnergren, H. Häffner, and N. Hermanspahn, Phys. Rev. A 62, 032510 (2000).

10. P. Kusch, S. Millman, and I. I. Rabi, Phys. Rev. 57, 765 (1940).

11. I. I. Rabi, Phys. Rev. 87, 379 (1952).

12. S. R. Lundeen and F. M. Pipkin, Phys. Rev. Lett. 46, 232 (1981).

13. S. R. Lundeen and F. M. Pipkin, Metrologia 22, 9 (1986). 
14. K. Grigoryev, R. Engels, F. Klehr, B. Lorentz, M. Mikirtytchiants, S. Mikirtytchiants, D. Prasuhn, F. Rathmann, J. Sarkadi, H. Seyfarth, H. Ströher, and A. Vasilyev, in Proceedings of the 17th International Spin Physics Symposium, edited by K. Imai, T. Murakami, N. Saito, and K. Tanida (AIP Conf. Proc. 915, 979, 2007).

15. R. Maier, Nucl. Instrum. Methods Phys. Res. Sect. A 464, 364 (2001).

16. R. Engels, R. Emmerich, J. Ley, G. Tenckhoff, M. Mikirtytchiants, F. Rathmann, H. Seyfarth, A. Vassiliev, and H. Paetz gen. Schieck, Rev. Sci. Instrum. 74, 4607 (2003).

17. R. Engels, R. Emmerich, K. Grigoriev, J. Ley, M. Mikirtytchiants, F. Rathmann, J. Sarkadi, H. Paetz gen. Schieck, H. Seyfarth, G. Tenckhoff, T. Ullrich, A. Vassiliev, Nucl. Instrum. Meth. in Phys. Res. Sect. A 536, 334 (2005).

18. J. L. McKibben, G. P. Lawrence, and G. G. Ohlsen, Phys. Rev. Lett. 20, 1180 (1968)

19. P. G. Sona, Energia Nucleare 14, 295 (1967).

20. P. Pradel, F. Roussel, A. S. Schlachter, G. Spiess, and A. Valance, Phys. Rev. A 10, 797 (1974).

21. I. N. Meshkov, Phys. At. Nucl. 61, 1679 (1998).

22. N. Kolachevsky, A. Matveev, J. Alnis, C. G. Parthey, S. G. Karshenboim, and T. W. Hänsch, Phys. Rev. Lett. 102, 213002 (2009).

23. N. Kolachevsky, M. Fischer, S. G. Karshenboim, and T. W. Hänsch, Phys. Rev. Lett. 92, 033003 (2004).

24. N. E. Rothery and E. A. Hessels, Phys. Rev. A. 61, 044501 (2000).

25. J. W. Heberle, H. A. Reich, and P. Kusch, Phys. Rev. 101, 612 (1956).

26. S. R. Lundeen, P. E. Jessop, and F. M. Pipkin, Phys. Rev. Lett. 34, 377 (1975). 
